\title{
Feynman-Hellmann Theorem and Signal Identification from Sample Covariance Matrices
}

\author{
Lucy J. Colwell, ${ }^{1}$ Yu Qin, ${ }^{1}$ Miriam Huntley, ${ }^{1}$ Alexander Manta, ${ }^{2}$ and Michael P. Brenner ${ }^{1}$ \\ ${ }^{1}$ School of Engineering and Applied Sciences and Kavli Institute for Bionano Science and Technology, \\ Harvard University, Cambridge, Massachusetts 02138, USA \\ ${ }^{2}$ Roche Diagnostics GmbH, Penzberg 82377, Germany \\ (Received 17 September 2013; revised manuscript received 6 July 2014; published 27 August 2014)
}

A common method for extracting true correlations from large data sets is to look for variables with unusually large coefficients on those principal components with the biggest eigenvalues. Here, we show that even if the top principal components have no unusually large coefficients, large coefficients on lower principal components can still correspond to a valid signal. This contradicts the typical mathematical justification for principal component analysis, which requires that eigenvalue distributions from relevant random matrix ensembles have compact support, so that any eigenvalue above the upper threshold corresponds to signal. The new possibility arises via a mechanism based on a variant of the FeynmanHellmann theorem, and leads to significant correlations between a signal and principal components when the underlying noise is not both independent and uncorrelated, so the eigenvalue spacing of the noise distribution can be sufficiently large. This mechanism justifies a new way of using principal component analysis and rationalizes recent empirical findings that lower principal components can have information about the signal, even if the largest ones do not.

DOI: 10.1103/PhysRevX.4.031032

\section{INTRODUCTION}

Technological advances enable the measurement of an ever increasing number of variables during an experiment. In biological settings, this occurs, for example, when measuring the expression levels of all genes in a cell at different time points or when comparing gene expression levels between individuals [1-3]. Examples from other fields include the time series of stock prices $[4,5]$, weather patterns [6], the study of networks [7,8], and so forth.

In these settings, the number of variables $p$ may be much larger than the number of measurements $n$. If $\mathbf{X}$ is the data set of $n$ column vectors, each of length $p$, then the sample covariance matrix is $\mathbf{C}=\mathbf{X} \mathbf{X}^{T} / n$. For large $n$, the sample covariance matrix converges to the true covariance matrix. However, when $p$ is of similar size, or larger than $n$, some correlations implied by the sample covariance matrix will be spurious [9-12].

A common technique for identifying true correlations among variables (signal) is principal component analysis. The largest eigenvalues, and so the largest principal components, are assumed to correspond to true correlations between sets of variables rather than spurious correlations caused by sampling noise. This means that the components of the corresponding eigenvectors are

Published by the American Physical Society under the terms of the Creative Commons Attribution 3.0 License. Further distribution of this work must maintain attribution to the author(s) and the published article's title, journal citation, and DOI.
Subject Areas: Statistical Physics

localized on these correlated sets of variables, which can be identified by, e.g., thresholding the top principal components [13-16].

The biological literature contains many recent examples where principal component analysis is applied (Fig. 1). In population genetics, the data consist of genetic polymorphisms across a large set of individuals and each principal component contains a coefficient for each individual. Those individuals with similarly distributed large coefficients on the top few principal components are found frequently to come from the same demographic population [17-21]. Finer-grained analysis of the location of individuals in biplots of principal components has revealed more detailed geographical delineations [18,22]. A similar approach identified correlated mutations within protein sequence alignments [23] by identifying groups of amino acids from biplots of the top few principal components; the different groups of amino acids were then found experimentally to control different properties of the protein.

When do outlying points on principal component biplots generate reliable hypotheses about sets of correlated variables? The typical model assumes the data matrix contains a signal added to background noise, where the background noise is assumed to be Gaussian, independent, and uncorrelated. Marčenko and Pastur (MP) proved that the eigenvalue distribution of the covariance matrix generated by this kind of background noise alone is bounded, with upper and lower limits that depend on the ratio of the number of variables $p$ to the number of samples $n$ [24]. Since the probability of an eigenvalue occurring outside these bounds due to sampling noise is very small 

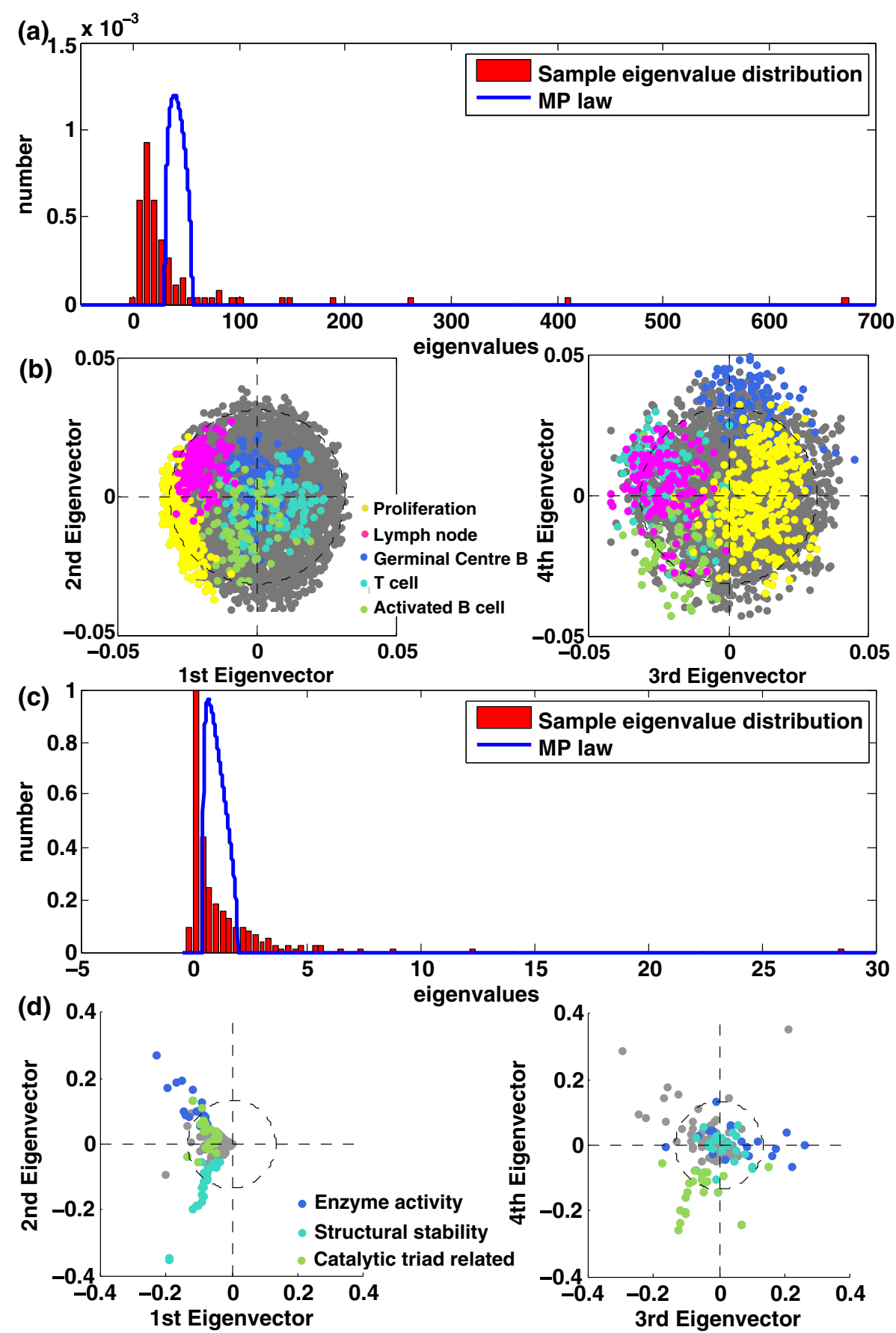

FIG. 1. (a),(b) Analysis of the lymphoma gene expression microarray data set [2], with 4026 genes measured under 96 different conditions. (a) The eigenvalue distribution of the covariance matrix (red histogram) is not well fit by the MP law (blue curve), as would be expected for signal superimposed on Gaussian, uncorrelated background noise. (b) Principal component biplots of 1st versus 2nd and 3rd versus 4th eigenvector components. The colored dots show the positions of clusters identified in the original analysis [2], corresponding to different cancer types. The different clusters show up as somewhat distinct regions in the principal component biplots. The dashed circle indicates a threshold on the eigenvector component size that would be expected for Gaussian, MP distributed background noise. The calibration of the circle radii is explained in the text. (c),(d) Analysis of a serine protease sequence alignment data set [23]. (c) Eigenvalue distribution compared with the MP law. (d) Typical principal component biplots. Colored points identify variables in clusters identified in Ref. [2].

$[9,12,15,25,26]$, any eigenvalue outside the bounds must be due to signal, meaning that the corresponding eigenvectors will have significant projection onto the subspace spanned by the signal variables. Such eigenvector components will be large and show up as outlier points on principal component biplots.

The present paper was motivated by peculiarities of applications of the Marčenko-Pastur distribution to signal 
identification in biological data sets. To give some context, Fig. 1 shows two examples of such data sets: Figs. 1(a) and 1(b) analyze a data set from a lymphoma gene expression microarray [2], measuring the expression levels of mRNA from 4026 genes in tissue samples from 96 different lymphocytes. Biological pathways naturally lead to dependencies between the production of different gene products, with different correlations between cells executing different programs, i.e., normal cells and those with different diseases. Reference [2] uses hierarchical clustering to discover that subtypes of lymphoma cancers can have different and distinctive gene expression patterns. But do the resulting correlations show up in a principal components analysis of such a data set? Since there are many more genes (4026) than samples (96), the covariance matrix of expression levels of different genes is undersampled and some of the measured correlations will be spuriously large. Figure 1(a) compares the eigenvalue distribution of the covariance matrix generated from this data with the Marčenko-Pastur distribution while Fig. 1(b) compares biplots of the largest principal components with the groups identified by Ref. [2]. Some of these groups appear in significant regions of the biplots (the contours indicate a significance level of $2 \sigma$ where $\sigma$ is the standard deviation expected for independent, identically distributed Gaussian noise) though it is not clear that these groups are clearly distinguished from other genes.

Figures 1(c) and 1(d) show a similar analysis from a different problem, concerning a set of protein sequences sampled from a family of proteins with similar structure and function (serine proteases) [23]. Here, Halabi et al. form a covariance matrix from a protein sequence alignment by measuring the frequency with which each pair of sequence positions in the alignment mutates together. Some correlated mutations occur by chance, while others occur because there is a functional or structural link between the two sequence positions within the protein. The AIM is to identify the groups of sequence positions that are functionally or structurally linked. The spectrum in Fig. 1(c) again shows a number of large eigenvalues outside the bulk of the Marčenko-Pastur distribution, while Fig. 1(d) shows that principal component biplots can identify groups of amino acids that correspond to the experimentally verified functional and structural groups.

These two examples both show the basic conundrum we address: how can we identify signal groups of variables directly from the biplots? From the biplots alone, the definitions of such groups seem somewhat arbitrary; ideally, a rigorous criterion should exist to determine whether a set of points is part of a group. A natural method is to compare the spectrum with the Marčenko-Pastur distribution and use this to identify the threshold of significant eigenvalues and corresponding eigenvectors. But in this context, Fig. 1 exposes two common peculiarities. First, the empirical eigenvalue distributions do not agree with the MP distribution. This removes the theoretical basis for a sharp eigenvalue threshold above which eigenvectors reflect signal, and not background noise. Most studies have dealt with this difficulty either heuristically or through empirical trial and error. For example, in the protein sequence alignment example [23], Halabi et al. randomly permute the samples for each variable, destroying all correlations in the data. The eigenvalue spectrum then agrees well with the MP distribution, making the upper threshold well defined. However, eigenvalues above this threshold that were caused by correlations between samples, i.e., shared ancestry, rather than signal correlations between variables have also been lost. In another approach, Patterson and Reich advocate changing the parameters of the MP distribution by decreasing the number of samples, to compensate for putative correlations between the different samples [17].

The second peculiarity is that the separation between outliers in principal component biplots can be much stronger for principal components with less than maximal eigenvalues, where these outliers correspond to groups of signal variables. This contradicts the basic idea behind using the MP distribution to identify the signal [e.g., eigenvectors 2 and 4 in Fig. 1(d)], since in the MP case the strength of the signal increases with distance of an eigenvalue above the threshold of the MP distribution.

Here, motivated by these observations, we show that even if the top principal components have coefficients of roughly uniform size, large coefficients on lower principal components can still correspond to a valid signal. This result arises from a different mechanism for a principal component to have a projection onto a signal, which is based on a variant of the celebrated Feynman-Hellmann theorem, invented for energy levels in quantum mechanics. This new mechanism applies even when the corresponding eigenvalue is below the upper threshold of the eigenvalue distribution of the background noise, and is especially relevant when the eigenvalue spacing of the noise distribution is sufficiently large and the spectrum differs substantially from the MP distribution, as occurs, for example, if there are correlations in the underlying noise distribution. Our analysis exploits the fact that the degree of alignment between the sample eigenvector corresponding to eigenvalue $\lambda_{i}(s)$ and the signal eigenvector depends on $d \lambda_{i} / d s$, where $s$ is the strength of the signal. Level repulsion between eigenvalues also affects the degree of eigenvector alignment, in a fashion similar to recent developments in quantum information theory [27]. Whether a signal can likely be recovered is determined by the ratio of the size of the random elements in the noise covariance matrix to the eigenvalue spacing of the full covariance matrix. The alignment of such eigenvectors with the signal most likely occurs when the corresponding eigenvalues are close to the signal eigenvalue, with the strength of the alignment being controlled by the ratio of the eigenvalue spacing to the size 
of typical elements in the sample covariance matrix. For a long-tailed eigenvalue distribution, the eigenvalue spacing within the tail is atypically large, making strong alignment possible.

\section{A MODEL PROBLEM}

We begin by analyzing a model problem. Here, the sample covariance matrix $\mathbf{M}$ is given by the sum of a signal matrix and a noise covariance matrix, i.e., $\mathbf{M}=\mathbf{W}+\mathbf{S}$, where $\mathbf{W}$ is the noise covariance matrix and $\mathbf{S}$ is the signal matrix, which has low rank. The mathematics is more straightforward in this case, but later we consider the case where the signal is an additive contribution to the noise matrix $\mathbf{X}$ rather than the noise covariance matrix $\mathbf{W}$, and the qualitative behavior is quite similar. For definiteness, we take $\mathbf{S}=s \mathbf{e}_{\mathbf{S}} \times \mathbf{e}_{\mathbf{S}}$, where $\mathbf{e}_{\mathbf{S}}$ is signal eigenvector and $s$ is a parameter that sets the signal strength.

We want to contrast two different cases, one where the eigenvalue distribution of $\mathbf{W}$ leads to the MP distribution, and the other in which it does not; we will see that the different shapes of the eigenvalue distributions in these cases lead to different signal detection properties. Generally speaking, the MP distribution occurs when the entries of the noise matrix are independent and uncorrelated [24]. We, thus, consider noise that is randomly distributed according to two types of distribution, the first of which satisfies these assumptions and the second of which does not.
Figure 2 shows the spectral characteristics of $\mathbf{W}=\mathbf{X} \mathbf{X}^{T} / n$, with the elements of $\mathbf{X}$ drawn independently from a Gaussian distribution, with $n=80$ samples (columns of $\mathbf{X}$ ) of $p=800$ variables. The spectrum of $\mathbf{M}$ is well fit by the MP distribution (blue line), while the first eigenvector is not Gaussian, enabling information about components of the signal (shown in green) to be identified. This information can be obtained by looking at principal component biplots. Figures 2(b)-2(d) show such biplots for different principal components; the information about which components are in the largest eigenvalue is clearly contained in the top eigenvector, though in none of the lower eigenvectors.

Figure 3 shows a contrasting situation where $\mathbf{X}$ is constructed to violate the assumptions of the MP distribution. A straightforward way of constructing such noise is to draw column vectors to be a Gaussian random vector, as in the case above, but then multiply each column by a different, randomly drawn weighting factor. Namely, if $\mathbf{X}=\left[\mathbf{x}_{1}, \mathbf{x}_{2}, \ldots, \mathbf{x}_{\mathbf{p}}\right]$, where each $\mathbf{x}_{\mathbf{i}}$ is a column vector of length $n$, then each $\mathbf{x}_{\mathbf{i}}=\eta_{i} \mathbf{y}_{\mathbf{i}}$, where $\mathbf{y}_{\mathbf{i}}$ is drawn from a multivariate Gaussian distribution and $\eta_{i}$ is a random number drawn from a different distribution. Here, we simply take the $\eta_{i}$ 's from a Gaussian distribution with unit variance. This model breaks the assumptions of the MP distribution because the entries of each column are distributed differently. This construction is introduced as a model for correlations among stock prices, where $\eta_{i}$

(a)

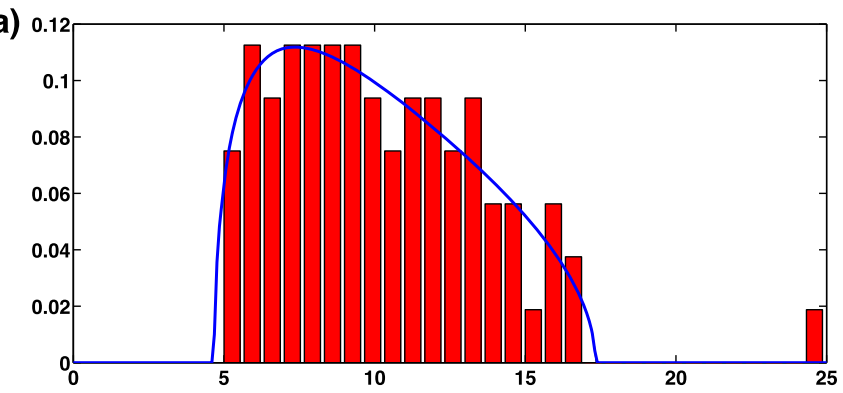

(b)

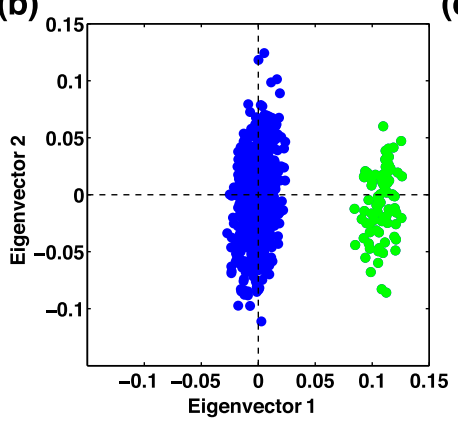

(c)

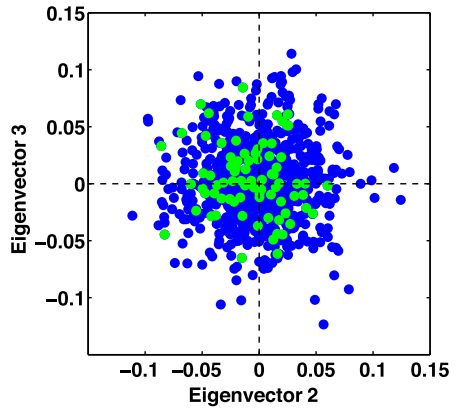

(d)

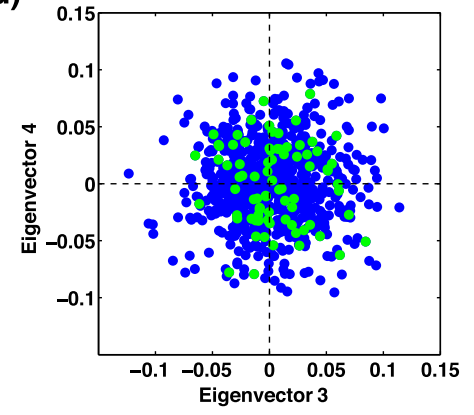

FIG. 2. Simulations where Gaussian distributed noise is superimposed upon a rank-1 signal perturbation. (a) The spectrum is well described by the MP distribution (blue curve), with a single eigenvalue that is far above the noise band. (b) Principal component biplot, showing the first eigenvector component plotted against the second, demonstrating that the magnitude of components in the first eigenvector captures the signal. Panels (c),(d) show that this information is not contained in the lower principal components; other lower principal component biplots are similar (data not shown). 


\section{(a)}

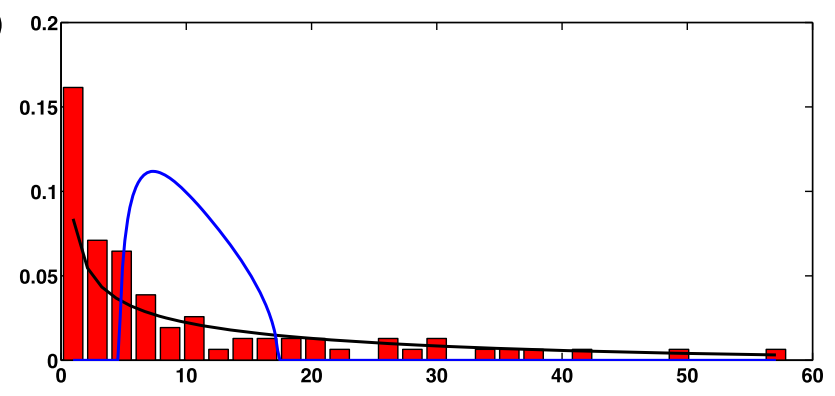

(b)

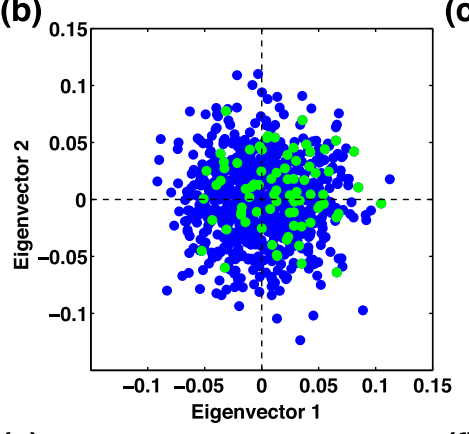

(e)

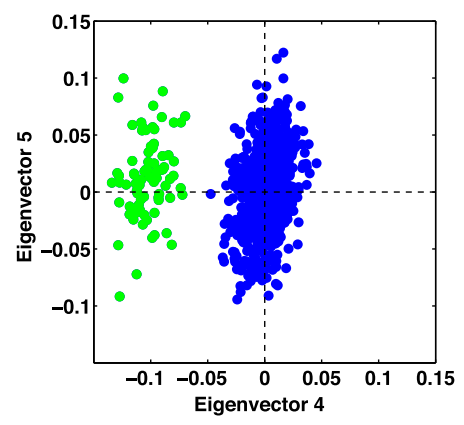

(c)

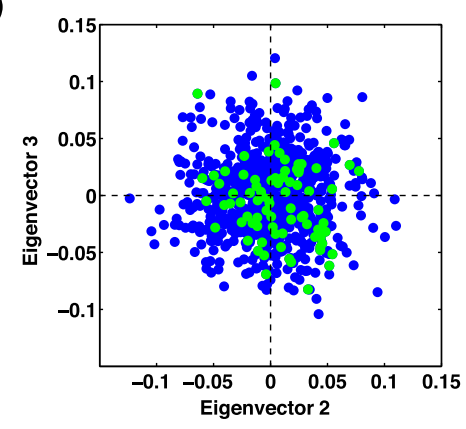

(f)

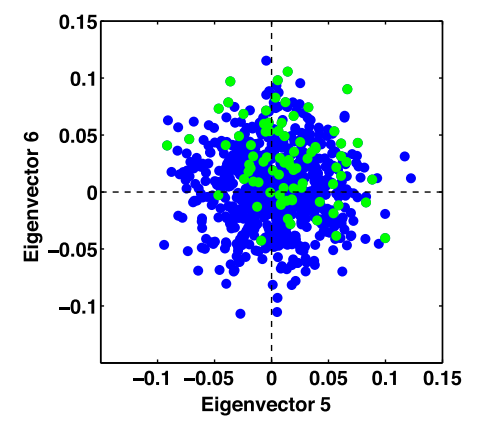

(d)

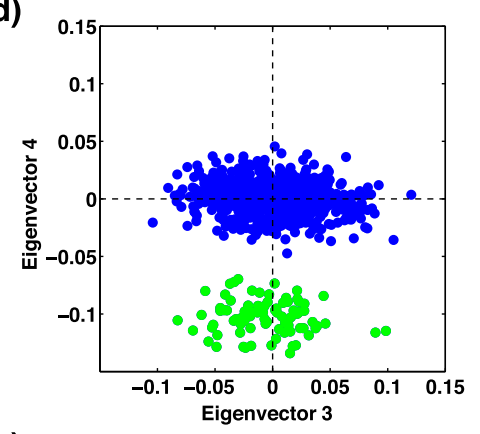

(g)

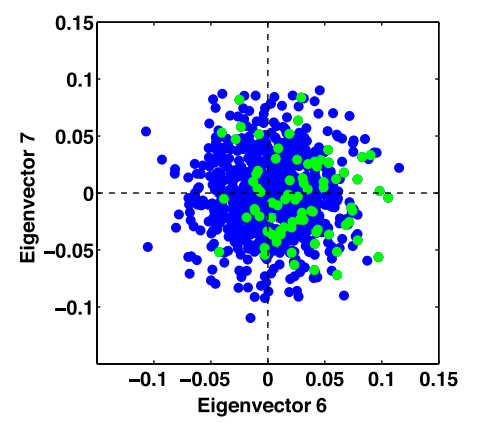

FIG. 3. Simulations where background noise that is not MP distributed is superimposed upon a rank-1 signal perturbation. The noise is generated by drawing $n=80$ vectors of length $p=800$ from a Gaussian distribution and then multiplying each vector by a (different) Gaussian random variable with unit variance. The signal strength $s=35.5$ is near the fourth eigenvalue. (a) The eigenvalue distribution does not agree with MP (blue curve), but is well described by the solution to a nonlinear integral equation for the density distribution (black curve); see the Appendix for details. (b),(c) Biplots of the first and second pairs of principal components do not identify the signal. (d),(e) However, the components of the fourth principal component clearly identify variables (shown in green) involved in the signal $s$. (f),(g) No information about the signal is present in the fifth, sixth, or seventh eigenvectors.

represents the time-dependent volatility, drawn from a distribution [5,28]. Although the model could serve as a potential model for the biological examples motivating this paper (e.g., if it were constrained to be positive definite, the $\eta_{i}$ could represent a normalization constant for different samples in a microarray), the main point of what follows is not the specific model-we show that if the assumptions underlying the derivation of the MP distribution are broken, there is a new possible mechanism for signal identification.

Figure 3 shows a number of striking features. Here, we tune the the signal strength $s$ to be $\approx 35$, near the fourth largest eigenvalue of the noise. First, as expected given the noise model, the eigenvalue distribution does not agree with the MP distribution [blue curve in Fig. 3(a)]. Because we have a generative model for the noise, it is still possible to derive the asymptotic shape of the distribution, using an integral equation also derived by Marčenko and Pastur. The result of this is given by the black curve in Fig. 3(a). Since the derivation is tangential to our main discussion, we include it in the Appendix. The most important feature of the distribution of eigenvalues is that it now has a long tail, in which the individual noise eigenvalues can be quite far apart, with a spacing that far exceeds the typical $n^{-1}$ characteristic of the MP distribution. The principal component biplots show a completely different phenomenology than that of Fig. 2. There is no trace of the signal in eigenvectors $1,2,3$ or 5,6,7-though there is a clear separation of the signal components on eigenvector 4 . Whereas the top few principal components do not contain information about variables involved in the signal, the 
fourth principal component does contain this information. This is the case even though the fourth eigenvalue contains only $\approx 7 \%$ of the total variance of the noise in the matrix, whereas the top three eigenvalues together capture $\approx 37 \%$.

\section{Feynman-Hellman mechanism}

The key to understanding these behaviors is the degree of alignment of $\mathbf{v}_{\mathbf{i}}(s)$, the $i$ th eigenvector of $\mathbf{M}$, with $\mathbf{e}_{\mathbf{S}}$. This follows from a variant of the celebrated FeynmanHellmann theorem [29]: Let $\lambda_{i}(s)$ denote the corresponding eigenvalue, so that

$$
\left(\mathbf{W}+s \mathbf{e}_{\mathbf{S}} \times \mathbf{e}_{\mathbf{S}}\right) \mathbf{v}_{\mathbf{i}}(\mathbf{s})=\lambda_{i}(s) \mathbf{v}_{\mathbf{i}}(\mathbf{s}) .
$$

Differentiating this equation with respect to $s$ gives

$$
\left(\mathbf{W}+s \mathbf{e}_{\mathbf{S}} \times \mathbf{e}_{\mathbf{S}}-\lambda_{i} \mathbf{I}\right) \frac{d \mathbf{v}_{\mathbf{i}}}{d s}=\frac{d \lambda_{i}}{d s} \mathbf{v}_{\mathbf{i}}(\mathbf{s})-\left(\mathbf{e}_{\mathbf{S}} \times \mathbf{e}_{\mathbf{S}}\right) \mathbf{v}_{\mathbf{i}}(s) .
$$

A solvability condition is required in order for a nonzero solution for $d \mathbf{v}_{\mathbf{i}} / d s$ to exist: This is because $\left(\mathbf{M}-\lambda_{i} \mathbf{I}\right) \mathbf{v}_{\mathbf{i}}=0$, so the right-hand side of Eq. (2) must be orthogonal to $\mathbf{v}_{\mathbf{i}}$. This implies the central result:

$$
\mathbf{v}_{\mathbf{i}} \cdot \mathbf{e}_{\mathbf{S}}=\sqrt{\frac{d \lambda_{i}}{d s}} .
$$

Note that this result is independent of the assumed noise distribution.

When $s \rightarrow \infty$, the largest eigenvalue of $\mathbf{M}, \lambda_{1} \approx s$, and Eq. (3) predicts that the largest eigenvector $\mathbf{v}_{1}(s)$ approaches perfect alignment with $\mathbf{e}_{\mathbf{S}}$. This explains Fig. 2, when the signal eigenvalue is outside the MP band. It is well known that in this case the largest eigenvector becomes aligned with $\mathbf{e}_{\mathbf{S}}$ as $s \rightarrow \infty$ [13].

The situation is more interesting in the case where $s$ is within the band of the noise eigenvalues (Fig. 3). As described above, those eigenvectors with corresponding eigenvalues closest to $s$ are somewhat aligned with the signal eigenvector $\mathbf{e}_{s}$ [Fig. 3(d)]. To understand how this can come about, we first consider the simple case where the matrix $\mathbf{W}$ is diagonal, with diagonal elements $d_{i}=2 i-1$, so $d_{1}<d_{2}<d_{3} \ldots<d_{n}$. At small $s$, the eigenvalues of $\mathbf{M}=\mathbf{W}+\mathbf{S}$ are then given exactly by $\lambda_{1}=d_{1}+s$ and $\lambda_{i}=d_{i}$, for $i>2$. As $s$ increases, the ordering of the eigenvalues switches, with the first eigenvalue $\lambda_{1}$ exceeding $\lambda_{i}$ when $s>d_{i}-d_{1}$. In Fig. 4(a), we plot $\lambda_{i}(s)$ for different values of $i$. The thick line highlights the change in the $j$ th eigenvalue of $\mathbf{M}$ with $s$. Equation (3) implies that initially the corresponding eigenvector is aligned with the $j-1$ st eigenvector of $\mathbf{W}$; with increasing $s$, it then switches to align with the signal eigenvector, and finally aligns with the $j$ th eigenvector. Therefore, the dot product $\mathbf{v}_{\mathbf{j}} \cdot \mathbf{e}_{\mathbf{S}}=1$ when $d_{j-1} \leq s \leq d_{j}$ and is zero otherwise.
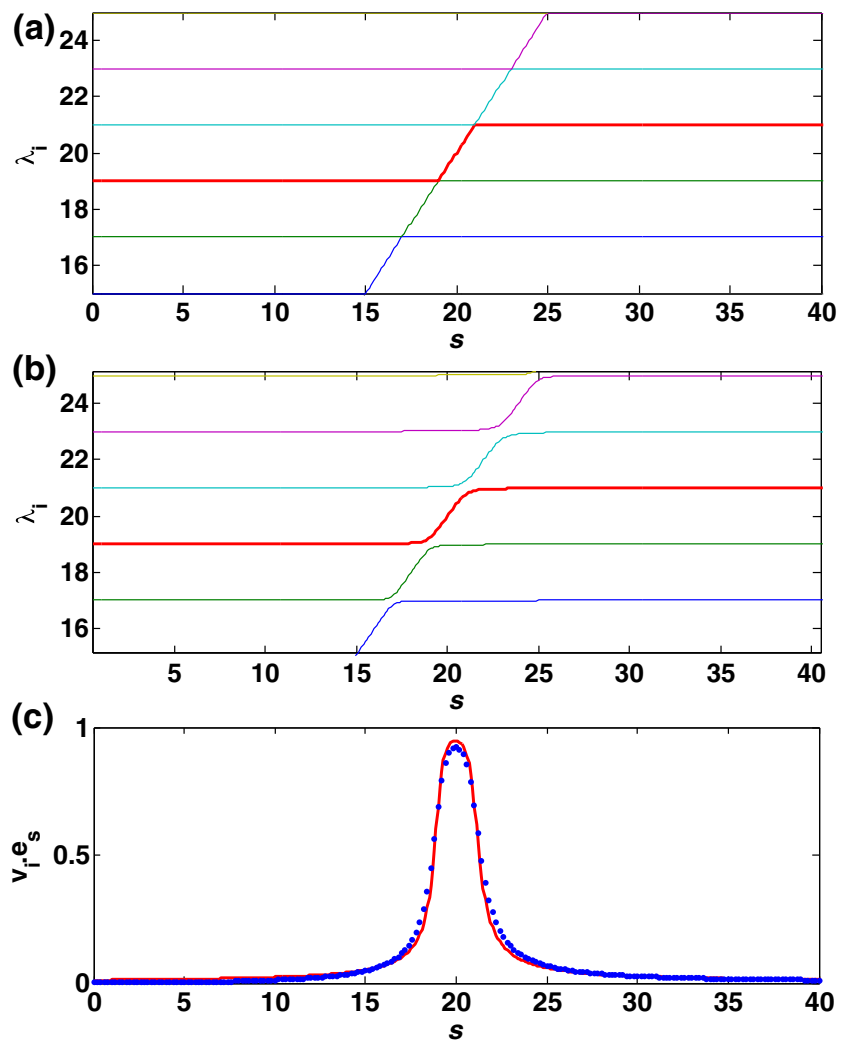

FIG. 4. (a) Eigenvalues of a diagonal matrix W, with eigenvalues $1,3,5, \ldots, 29$, perturbed by the matrix $\mathbf{S}$, as a function of signal strength $s$. As $s$ increases, different eigenvectors become parallel to the signal eigenvector. The red curve shows the typical behavior of an eigenvalue with increasing $s$. (b) As (a), except here $\mathbf{W}$ is no longer diagonal: $W(i, 1)=W(1, i)=\epsilon_{i}$, where $\epsilon_{i}$ are random numbers with $0 \leq \epsilon_{i} \leq 0.5$. Note the "level repulsion" now visible between the different eigenvalues, so that the red curve continuously varies from 19 to 21. (c) Eigenvector alignment $\mathbf{v}_{\mathbf{i}} \cdot \mathbf{e}_{\mathbf{S}}$ corresponding to the red curve in (b) (red curve). The blue dots correspond to the theory of Eq. (7).

If the matrix $\mathbf{W}$ is not diagonal, then the Wigner-von Neumann theorem states that eigenvalues of different levels cannot cross [30]. Indeed, this behavior is illustrated by introducing $W(1, i)=W(i, 1)=\epsilon_{i}$, for $1<i \leq n$. Figure 4(b) shows how the eigenvalues vary with $s$ for random $\epsilon_{i}$ drawn uniformly in $\left[0, \frac{1}{2}\right]$. The eigenvalue curves (e.g., the red solid curve) no longer intersect. Correspondingly, $\mathbf{v}_{\mathbf{i}} \cdot \mathbf{e}_{\mathbf{S}}$ is a continuous curve, with a peak value smaller than unity [Fig. 4(c)].

The maximum alignment of $\mathbf{v}_{\mathbf{i}} \cdot \mathbf{e}_{\mathbf{S}}$ can be calculated by noting that each $\lambda_{i}(s)$ exhibits two different level repulsions: At small $s, \lambda_{i}(s)$ corresponds to the eigenvalue $d_{j-1}$, with eigenvector $\mathbf{v}_{\mathbf{j}-\mathbf{1}}$. This transitions when $s \approx d_{j-1}$ to the eigenvalue $\lambda_{i}(s) \approx s$, with eigenvector $\mathbf{e}_{\mathbf{S}}$. Finally, for $s>d_{j}, \lambda_{i}(s) \rightarrow d_{j}$, with eigenvector $\mathbf{v}_{\mathbf{j}}$. The first level repulsion occurs at the transition from $d_{j-1} \rightarrow s$, whereas the second level repulsion occurs at the transition from $s \rightarrow d_{j}$. 
Each of these transitions can be separately analyzed by considering the reduced matrix $\mathbf{M}^{\text {red}}$, which controls the transitions, namely, for the first transition:

$\mathbf{M}^{\mathrm{red}}=\left(\begin{array}{cc}\mathbf{e}_{\mathbf{s}} \mathbf{M} \mathbf{e}_{\mathbf{s}} & \mathbf{e}_{\mathbf{s}} \mathbf{M} \mathbf{v}_{\mathbf{j}-\mathbf{1}} \\ \mathbf{e}_{\mathbf{s}} \mathbf{M} \mathbf{v}_{\mathbf{j}-\mathbf{1}} & \mathbf{v}_{\mathbf{j}-\mathbf{1}} \mathbf{M} \mathbf{v}_{\mathbf{j}-\mathbf{1}}\end{array}\right)=\left(\begin{array}{cc}s & \epsilon_{j-1} \\ \epsilon_{j-1} & d_{j-1}\end{array}\right)$.

The eigenvalue of $\mathbf{M}^{\text {red }}$ that corresponds to the transition from $d_{j-1} \rightarrow s$ then corresponds to

$$
\lambda_{<}(s)=\frac{1}{2}\left(s+d_{j-1}+\sqrt{\left(s-d_{j-1}\right)^{2}+4 \epsilon_{j-1}^{2}}\right) .
$$

Similarly, the transition in eigenvalue from $s \rightarrow d_{j}$ is described by

$$
\lambda_{>}(s)=\frac{1}{2}\left(s+d_{j}-\sqrt{\left(s-d_{j}\right)^{2}+4 \epsilon_{j}^{2}}\right) .
$$

When $\epsilon_{j}, \epsilon_{j-1}$ are much smaller than the level spacing $d_{j}-d_{j-1}$, the eigenvalue transition is well captured by the composite formula $\lambda_{i}(s) \approx \lambda_{<}(s)+\lambda_{>}(s)-s$. Hence, the eigenvector alignment is given by

$$
\mathbf{v}_{\mathbf{i}} \cdot \mathbf{e}_{\mathbf{S}}=\sqrt{\frac{d \lambda_{<}}{d s}+\frac{d \lambda_{>}}{d s}-1} .
$$

Figure 4(c) compares the predictions of this formula to the numerical simulations, with excellent agreement. If we assume that $\epsilon=\epsilon_{j}=\epsilon_{j-1}$, we obtain a particularly simple formula for maximum degree of eigenvector alignment, namely,

$$
\max _{s}\left(\mathbf{v}_{\mathbf{i}} \cdot \mathbf{e}_{\mathbf{S}}\right)^{2}=\frac{\Delta}{\sqrt{\Delta^{2}+16 \epsilon^{2}}},
$$

where $\Delta=d_{j}-d_{j-1}$ is the level spacing. The maximum alignment is determined by the ratio of $\Delta$ to the magnitude of the off-diagonal elements $\epsilon$. When $\Delta \gg \epsilon$, level repulsion is very weak, and $\mathbf{v}_{\mathbf{i}} \cdot \mathbf{e}_{\mathbf{S}} \approx 1$; in contrast, when $\Delta \ll \epsilon$, the level repulsion dominates the transition in eigenvalues from $d_{j-1} \rightarrow d_{j}$, and the alignment $\mathbf{v}_{\mathbf{i}} \cdot \mathbf{e}_{\mathbf{S}} \ll 1$. Note also that $\Delta$ sets the range of $s$, where the eigenvector alignment can be significant. This means that for every signal eigenvalue, we expect only those sample eigenvectors that correspond to nearby sample eigenvalues to align with the signal eigenvector.

These results now easily generalize to the case where $\mathbf{W}$ is a random matrix. Figure 5(a) shows the evolution of a few eigenvalues of a heavy-tailed random matrix with signal strength $s$, showing a similar phenomenology to the model problem: the eigenvalues transition from one constant at small $s$ to another constant at large $s$. The constants at small $s$ are just the eigenvalues $\lambda_{i}^{0}$ of $\mathbf{W}$, with eigenvectors $\mathbf{u}_{\mathbf{i}}^{\mathbf{0}}$. At large $s$, other than the top eigenvalue $(\approx s)$, the constants are the eigenvalues of the submatrix of $\mathbf{W}$ that is orthogonal to $\mathbf{e}_{\mathbf{S}}$. When $\mathbf{e}_{\mathbf{S}}=[1, \mathbf{0}]$, this matrix is obtained by deleting the first row and column of $\mathbf{W}$.

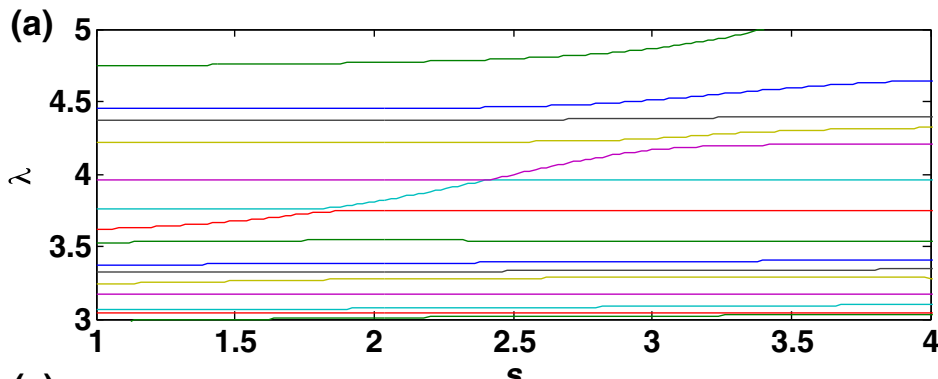

(c)

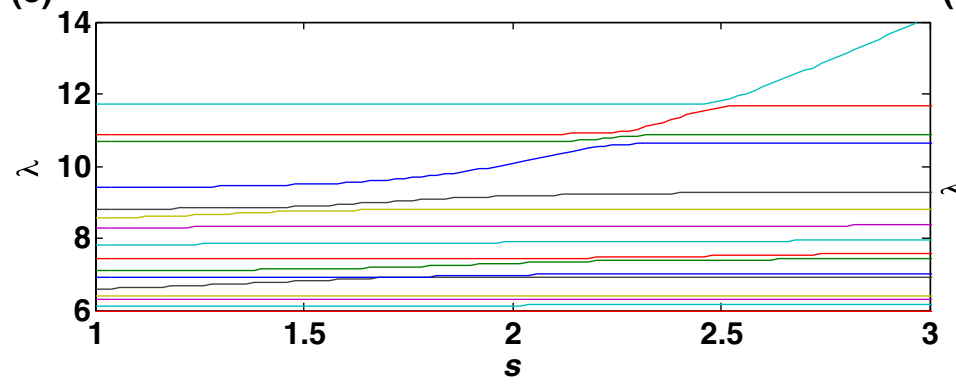

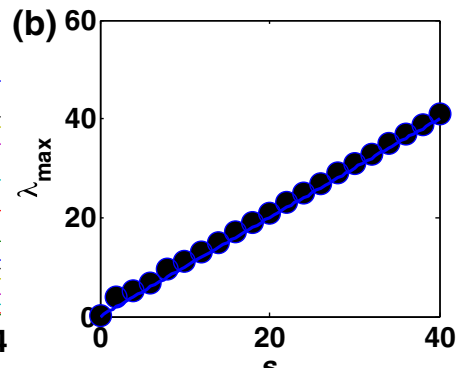

(d)

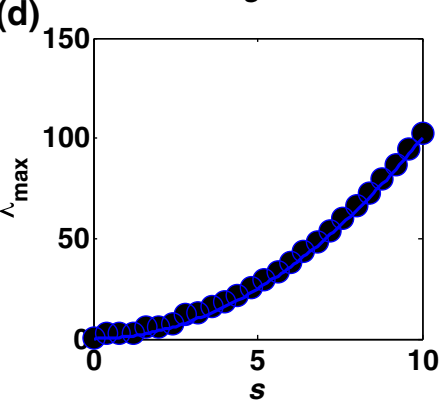

FIG. 5. (a) Eigenvalues of a random matrix $\mathbf{M}=\mathbf{W}+\mathbf{S}$ as a function of signal strength $s$. Note the same qualitative characteristics of the eigenvalue as that for the matrix in Fig. 4. (b) Location of eigenvalue where the maximum eigenvector alignment occurs for $\mathbf{M}=\mathbf{W}+\mathbf{S}$, as a function of signal strength, with excellent agreement with the $\lambda=s$ law (solid line). (c) Eigenvalues of $\mathbf{M M}^{T}$, where $\mathbf{M}=\mathbf{X}+\mathbf{S}$, with $\mathbf{X}$ a $p \times n$ noise matrix, as a function of $s$. Note the same qualitative structure as the other cases. (d) Location of eigenvalue where the maximum eigenvector alignment occurs for $\mathbf{M}=\mathbf{X}+\mathbf{S}$, as a function of signal strength, with excellent agreement with the $\lambda=s^{2}$ law (solid line). 
We denote the eigenvalues and eigenvectors of this submatrix by $\lambda_{i}^{\infty}, \mathbf{u}_{\mathbf{i}}^{\infty}$.

The analysis of this random matrix problem proceeds exactly as above: in the reduced matrix $\mathbf{M}^{\text {red }}$ [cf. Eq. (4)], the vector $\mathbf{v}_{\mathbf{j}-\mathbf{1}}$ corresponds to the eigenvector either before (when $s$ is small) or after (when $s$ is large) the transition. As above, the alignment of the eigenvector depends on the ratio of the eigenvalue spacing $\Delta=\lambda_{i}^{\infty}-\lambda_{i}^{0}$ to the off-diagonal elements $\epsilon_{j}=\mathbf{e}_{\mathbf{S}} \mathbf{M}\left(s^{*}\right) \mathbf{u}_{\mathbf{i}}^{\infty}$ and $\epsilon_{j-1}=\mathbf{e}_{\mathbf{S}} \mathbf{M}\left(s^{*}\right) \mathbf{u}_{\mathbf{i}}^{0}$, where in both cases $\mathbf{M}$ is evaluated at $s^{*}$, the value of $s$ where the transition occurs. A calculation implies that $\epsilon_{j-1}=$ $s u_{1,1}^{0}+\mathbf{w}_{1} \cdot \mathbf{u}_{1}^{\mathbf{0}}$, where $\mathbf{w}_{1}$ is the first column of $\mathbf{W}$. Since both $u_{1,1}^{0}, w_{1, j}$ are $O(1 / \sqrt{n})$, we have that $\epsilon_{j-1} \sim O(s / \sqrt{n})$. A similar result holds for $\epsilon_{j}$, so that $\epsilon \sim(s / \sqrt{n})$.

The relative size of $\epsilon$ and the eigenvalue spacing $\Delta$ determines the degree of eigenvector alignment with the signal. Within the Marčenko-Pastur or Wigner bands, $n$ eigenvalues are confined to a finite interval, so $\Delta \sim O(1 / n)$. Thus, the degree of eigenvector alignment will be weak. On the other hand, within a long tail, the level spacing will be larger, and thus strong alignment of nearby sample eigenvectors with the signal eigenvector can occur. As a test of these calculations, Fig. 5(b) shows the eigenvalue where the maximum eigenvector alignment occurs, as a function of signal strength, for a single realization of $\mathbf{W}$. The agreement with the level crossing law $\lambda=s$ is excellent.

\section{PERTURBING THE DATA MATRIX}

Thus far, we have analyzed the model $\mathbf{M}=\mathbf{W}+\mathbf{S}$, in which a signal is added to the noise covariance matrix. The case where the signal is added directly to the data (in this case noise) matrix follows by close analogy. As above, we consider a noise matrix $\mathbf{X}$, which has $p$ rows representing different variables (e.g., genes) and $n$ columns representing different samples (e.g., time points or conditions). We consider a signal matrix $s \mathbf{S}$, where $n^{-1}\left(\mathbf{S S}^{T}\right)$ is rank 1 , with eigenvector $\mathbf{e}_{\mathbf{S}}$ and eigenvalue $\lambda_{S}$. The covariance matrix is given by $\mathbf{M}=n^{-1}(\mathbf{X}+s \mathbf{S})(\mathbf{X}+s \mathbf{S})^{T}$. The qualitative phenomenology is identical to the case described above: Figure 5(c) shows the evolution of the eigenvalues of $\mathbf{M}$ as a function of the signal strength $s$ : as above, the eigenvalues transition from a constant at small $s$ (given by the eigenvalues of $\mathbf{X} \mathbf{X}^{T}$ ) to a different constant for large $s$ (given by the eigenvalues of the submatrix of $\mathbf{X} \mathbf{X}^{T}$ that is orthogonal to $\mathbf{e}_{\mathbf{S}}$ ).

There are two main qualitative differences between the present case and the case analyzed above: First, the relevant parameter controlling the strength of the signal is $s^{2}$ instead of $s$. This changes both the shape of the eigenvalue curves when viewed as a function of $s$ as well as the locations of the transitions. Transitions from an eigenvalue of the random matrix to that of the signal occur when $\lambda=s^{2}$; moreover, when a particular eigenvalue curve is dominated by the signal eigenvector, its shape follows $\lambda=s^{2}$. These are both in contrast to the previous case, where both the shape of the eigenvalue curve and transitions followed $\lambda=s$ [Fig. 5(b)]. Figure 5(d) shows the eigenvalue where the maximum eigenvector alignment occurs as a function of signal strength, for a single realization of $\mathbf{X}$. The agreement with the level crossing law $\lambda=s^{2}$ is excellent.

Second, the different form of $\mathbf{M}$ complicates the connection between $\mathbf{v}_{\mathbf{i}} \cdot \mathbf{e}_{\mathbf{S}}$ and $d \lambda_{i} / d s$ : Repeating the derivation above for the present case, we differentiate the equation $n^{-1}\left[\mathbf{X} \mathbf{X}^{T}+2 s\left(\mathbf{S X}^{T}+\mathbf{X S}^{T}\right)+s^{2} \mathbf{S S}^{T}\right] \mathbf{v}_{\mathbf{i}}(\mathbf{s})=\lambda_{i}(s) \mathbf{v}_{\mathbf{i}}(\mathbf{s})$, with respect to $s$, implying

$$
\begin{gathered}
n^{-1}\left[\mathbf{X} \mathbf{X}^{T}+2 s\left(\mathbf{S X}^{T}+\mathbf{X S}^{T}\right)+s^{2} \mathbf{S S}^{T}-\lambda_{i} \mathbf{I}\right] \frac{d \mathbf{v}_{\mathbf{i}}}{d s} \\
=\frac{d \lambda_{i}}{d s} \mathbf{v}_{\mathbf{i}}(\mathbf{s})-n^{-1}\left(2 \mathbf{S} \mathbf{X}^{T}+2 \mathbf{X S}^{T}+2 s \mathbf{S} \mathbf{S}^{T}\right) \mathbf{v}_{\mathbf{i}}(s) .
\end{gathered}
$$

Again, we need a solvability condition for the equation to have a solution for $d \mathbf{v}_{\mathbf{i}} / d s$ : the right-hand side of the equation must be orthogonal to $\mathbf{v}_{\mathbf{i}}$. This then implies that

$$
\mathbf{v}_{\mathbf{i}} \cdot\left(2 n^{-1} \mathbf{S} \mathbf{X}^{T}+2 n^{-1} \mathbf{X} \mathbf{S}^{T}+2 s n^{-1} \mathbf{S S}^{T}\right) \mathbf{v}_{\mathbf{i}}(s)=\frac{d \lambda_{i}}{d s} .
$$

This equation can be turned into an approximate relationship for $\mathbf{v}_{\mathbf{i}} \cdot \mathbf{e}_{\mathbf{S}}$, by noting that (when $s$ is at least order unity) $\mathbf{v}_{\mathbf{i}} \cdot\left(n^{-1} \mathbf{S} \mathbf{X}^{T}+n^{-1} \mathbf{X} \mathbf{S}^{T}\right) \mathbf{v}_{\mathbf{i}}$ is small compared to $2 s \mathbf{v}_{\mathbf{i}} n^{-1} \mathbf{S S}^{T} \mathbf{v}_{\mathbf{i}}=2 \lambda_{S}\left(\mathbf{v}_{\mathbf{i}} \cdot \mathbf{e}_{\mathbf{S}}\right)^{2}$. Thus, we arrive at the approximate condition

$$
2 s \lambda_{S}\left(\mathbf{v}_{\mathbf{i}} \cdot \mathbf{e}_{\mathbf{S}}\right)^{2} \approx \frac{d \lambda_{i}}{d s}
$$

Figure 6 compares this approximation with a simulation, in which $n=400, p=200$, and $\mathbf{S S}^{T}$ is rank 1 . The noise is generated as described above for the simulations in Fig. 3; each sample (drawn from a multivariate normal) is multiplied by a Gaussian random variable. The approximation works quite well in the regime of interest, capturing both the shape and the location of the eigenvector alignment. The discrepancy at small $s$ occurs when this approximation breaks down.

These remarks show that the basic mathematical features that govern low-rank perturbations to a covariance matrix also apply when the perturbation is applied to the data matrix. The degree of eigenvalue alignment with the signal again depends on the ratio of level spacing to the offdiagonal elements $(\epsilon)$ as defined in Eq. (4), and a straightforward calculation shows that $\epsilon \sim 1 / \sqrt{n}$, exactly as above. This means that a high degree of eigenvector alignment within the noise band requires that the eigenvalue spacing is larger than $1 / \sqrt{n}$, requiring that the spectrum of the noise has a long tail. 


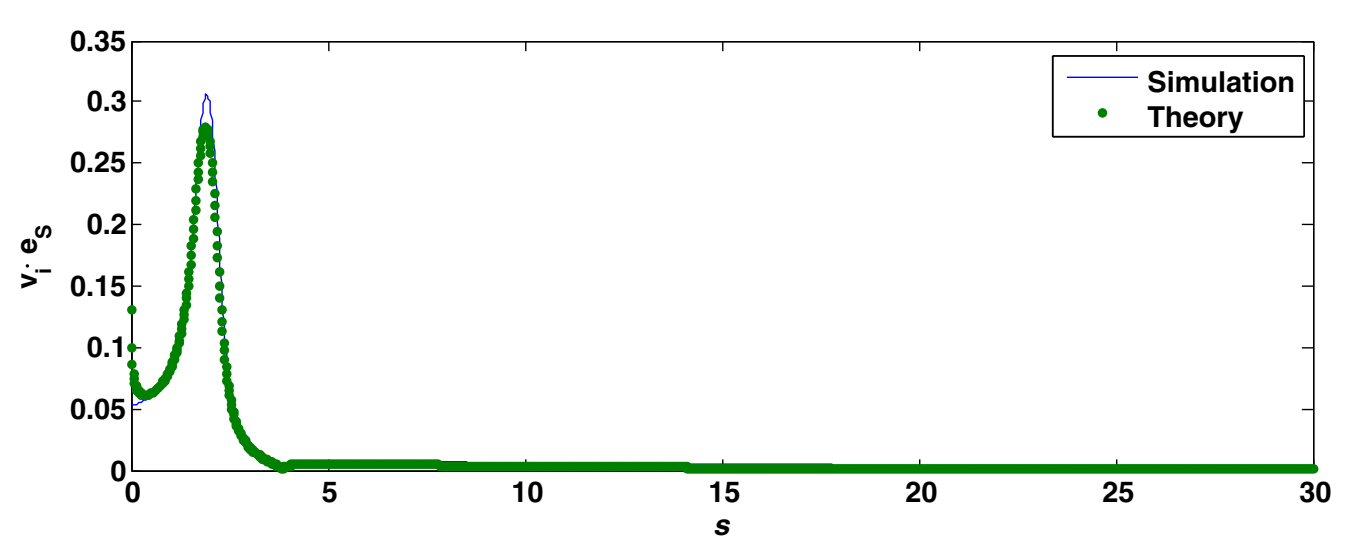

FIG. 6. Comparison of $\mathbf{v}_{\mathbf{i}} \cdot \mathbf{e}_{\mathbf{S}}$ with Eq. (11). Here, $\mathbf{X}$ has $n=400$ samples of $p=200$ variables, as discussed in the text, with signal matrix $s \mathbf{S}$, where $\mathbf{S S}^{T}$ is rank 1 .

\section{DISCUSSION}

A common method for extracting true correlations from large data sets is to look for groups of outlying points in biplots of principal components with the largest eigenvalues. This is typically justified using the fact that the eigenvalue distribution of the covariance matrix of a matrix drawn with random entries has compact support (e.g., the Wigner or MP distributions), so that any eigenvalue above the upper threshold must correspond to signal $[9,12,15]$. In this paper, we expose another logical possibility, which occurs for background noise distributions that do not have compact support and, in particular, where the eigenvalue distribution has a long tail.

We demonstrate that the eigenvector $\mathbf{v}_{\mathbf{i}}$ of a sample covariance matrix can have a significant degree of alignment with a signal eigenvector $\mathbf{e}_{\mathrm{S}}$ even when the eigenvalue corresponding to the signal is within the range of the noise eigenvalues. This suggests that lower eigenvalues can have information about the signal, even if the largest ones do not. Our analysis shows that alignment of $\mathbf{v}_{\mathbf{i}}$ with $\mathbf{e}_{\mathbf{S}}$ most likely occurs for eigenvalues of the sample covariance matrix that are close to the signal eigenvalue; the strength of the alignment is then controlled by the ratio of the eigenvalue spacing to the size of typical elements in the noise matrix (e.g., $1 / \sqrt{n}$ ). Fulfillment of this condition is quite difficult within the (bounded) eigenvalue spectrum of MP distributed noise, where typically the level spacing is $O\left(n^{-1}\right)$. However, within a long-tailed distribution, as occurs when the conditions for the MP distribution (independent and uncorrelated noise) are not obeyed, the eigenvalue spacing can be much larger, making such alignment possible.

What approach should be taken in practice? Our analysis gives a mathematical justification for seeking outlier groups of principal components. Even when the top principal components do not show any such correlations, the mechanism described in this paper implies that the lower ones can, at least when the eigenvalue spacings in the noise distribution are sufficiently large. These conditions appear to hold in the data sets shown in Fig. 1. Both the microarray measurements and the serine protease sequence alignment have eigenvalue distributions that differ substantially from the Marčenko-Pastur distribution; in particular, they each have a much longer tail. At the same time, variables that were identified as being part of the "signal" form outlying groups in biplots of lower principal components.

Of course, to show definitively that the mechanism here applies requires that there is a generative model for the noise distribution, which we usually do not have. Therefore, in practice, some amount of empiricism is called for in identifying the eigenvalue threshold above which eigenvector component correlations can be significant. Our analysis demonstrates that correlations between eigenvector components can occur at a different value than that suggested by the traditional threshold implied by the Marčenko-Pastur distribution, where the precise threshold depends on the nature of the eigenvalue distribution of the noise.

\section{ACKNOWLEDGMENTS}

We thank Martin Erkens, Florian Lipsmeier, Anton Belousov, and Sue Coppersmith for important conversations. This research was supported by Roche Pharmaceuticals. L.C. was supported by EPSRC (EPH028064/2) and thanks the Institute for Advanced Study, Princeton, for their hospitality and support while this paper was being written. M. P. B. acknowledges support from the Simons Foundation.

\section{APPENDIX}

Random matrix theory is rich with studies examining the limiting spectral distributions of various types of random matrices, the most famous example perhaps being the semicircle law for Wigner matrices. The study of sample covariance matrices began with the seminal work of Marčenko and Pastur [24] who derived an integral equation 
for their limiting eigenvalue distributions. The limiting distribution of a sample covariance matrix depends, unsurprisingly, on the form of the true covariance matrix of the variables. The classical Marčenko-Pastur solution corresponds to the case where the variables are identically and independently distributed, i.e., the true covariance matrix is the identity matrix. In this case, the integral equation has an analytical solution, the so-called Marčenko-Pastur distribution. However, the original equation of Marčenko and Pastur is valid even in the case of nontrivial correlations between the variables. For completeness, we include the derivation here, as well as comments on how we solve this equation numerically to obtain the black curve in Fig. 3(a).

As an important aside, we note that the literature that examines spectra of sample covariance matrices often frames the discussion in terms of $p$ variables, which are correlated, and $n$ samples, which are uncorrelated. That is, the goal is often to derive or bound the spectrum of an empirical covariance matrix of the variables, given the true covariance matrix. In this paper, we demonstrate that the spectrum of the empirical covariance matrix of the variables can change dramatically as a result not of correlations between variables but rather correlations between samples, which mean that the background noise is not MP distributed. The distinction, in fact, is purely semantic, as the Marčenko-Pastur integral equation is valid in either case. The spectrum of $X X^{T}$ is identical to that of $X^{T} X$ (with the exception of delta function centered at zero in the spectral distribution of the larger of the two matrices). In order to remain consistent with previous literature, in the following derivation, we retain the language of the interesting correlations occurring between the $p$ variables, and keep the $n$ samples uncorrelated.

To start, we need to define the Stieltjes transform. Given a spectral distribution $f(\lambda)$, we define its Stieltjes transform

$$
m_{f}=\int \frac{f(\lambda) d \lambda}{\lambda-z}
$$

defined for all $z$ in the upper complex plane. The Stieltjes transform contains the eigenvalue distribution, through the inversion formula

$$
f(\lambda)=\lim _{\epsilon \rightarrow 0} \frac{1}{\pi} \operatorname{Im}\left[m_{f}(x+i \epsilon)\right] .
$$

With this definition, we can write the Marčenko-Pastur equation as, e.g., derived by Silverstein and Bai [31]. Let us assume that the data matrix $X$ is an $n$ by $p$ matrix that can be written as $X=Y \Sigma_{p}^{1 / 2}$, where the entries of $Y$ are independent and identically distributed and each element of $Y$ has zero mean and unit variance. $\Sigma_{p}$ is a $p \times p$ positive definite matrix and can be considered the "true" covariance matrix of the $p$ variables.
Define $\gamma=p / n$. Further, we assume that $H_{p}$ is the spectral distribution of $\Sigma_{p}$ and assume that $H_{p}$ converges weakly to the distribution $H_{\infty}$. Finally, denote $f_{p}$ as the spectral distribution of the $X X^{T} / n$, and let $m_{f_{p}}$ be its Stieltjes transform.

Then, Marčenko and Pastur proved that $m_{f_{p}}$ converges to $m_{\infty}$ as $p \rightarrow \infty$ with $\gamma$ fixed, where $m_{\infty}$ obeys

$$
-\frac{1}{m_{\infty}(z)}=z-\gamma \int \frac{\lambda d H_{\infty}(\lambda)}{1+\lambda m_{\infty}(z)}
$$

for all $z$ in the upper complex plane.

Using this equation, we can numerically solve for the Stieltjes transform for any $H_{\infty}$. To do so, we break the equation into its real and imaginary parts and discretize in $z$ [32]. We then solve for $m_{\infty}(z)$ using the MATLAB function fsolve. The numerical Stieltjes transform can then be inverted to obtain the limiting spectral distribution of the sample covariance matrix.

In the main text, we describe a simple model where all of the noise columns are multiplied by random numbers drawn from the same distribution; in Fig. 3(a), we employ a Gaussian distribution with mean zero and unit variance, but, in general, this distribution could be any reasonable probability density function $r(x)$. Multiplying the noise columns amounts to, in the notation used above, a diagonal $\Sigma_{p}$ matrix, whose diagonal entries are the square of variables drawn from $r(x)$. To solve the MarčenkoPastur integral equation, we need the limiting spectral distribution of $\Sigma_{p}$. In this case, deriving such a distribution is simple, as it is just given by the distribution of the diagonal entries. Thus, $H_{\infty}(x)=\left(r\left(x^{1 / 2}\right)+r\left(-x^{1 / 2}\right)(/ 2 \sqrt{x}\right.$ for $x>0$. By inputting this function into the integral equation, we can extract the resulting spectral distribution of the empirical covariance matrix [see black curve in Fig 3(a)].

[1] J. K. Pickrell, J. C. Marioni, A. A. Pai, J. F. Degner, B. E. Engelhardt, E. Nkadori, J. B. Veyrieras, M. Stephens, Y. Gilad, and J.K. Pritchard, Understanding Mechanisms Underlying Human Gene Expression Variation with RNA Sequencing, Nature (London) 464, 768 (2010).

[2] A. A. Alizadeh, M. B. Eisen, R. E. Davis, C. Ma, I. S. Lossos, A. Rosenwald, J. C. Boldrick, H. Sabet, T. Tran, and X. Yu, Distinct Types of Diffuse Large B-Cell Lymphoma Identified by Gene Expression Profiling, Nature (London) 403, 503 (2000).

[3] L. D. W. Hillier, V. Reinke, P. Green, M. Hirst, M. A. Marra, and R. H. Waterston, Massively Parallel Sequencing of the Polyadenylated Transcriptome of C. Elegans, Genome Res. 19, 657 (2009).

[4] J.-P. Bouchaud, L. Laloux, M. A. Miceli, and M. Potters, Large Dimension Forecasting Models and Random Singular Value Spectra, Eur. Phys. J. B 55, 201 (2007). 
[5] J.-P. Bouchaud and M. Potters, Financial Applications of Random Matrix Theory: A Short Review, arXiv:0910.1205.

[6] M. S Santhanam and P. K. Patra, Statistics of Atmospheric Correlations, Phys. Rev. E 64, 016102 (2001).

[7] R. R. Nadakuditi and M. E. J. Newman, Graph Spectra and the Detectability of Community Structure in Networks, Phys. Rev. Lett. 108, 188701 (2012).

[8] R. R. Nadakuditi and M. E. J. Newman, Spectra of Random Graphs with Arbitrary Expected Degrees, Phys. Rev. E 87, 012803 (2013).

[9] I. M. Johnstone, in Proceedings of the International Congress of Mathematicians, Madrid, 2006 (Asociación International Congress of Mathematicians, Madrid, 2006), pp. 307-333.

[10] I. M. Johnstone and D. M. Titterington, Statistical Challenges of High-Dimensional Data, Phil. Trans. R. Soc. A 367, 4237 (2009).

[11] Z. Burda, A. Görlich, A. Jarosz, and J. Jurkiewicz, Signal and Noise in Correlation Matrix, Physica (Amsterdam) 343A, 295 (2004).

[12] N. El Karoui, Recent Results about the Largest Eigenvalue of Random Covariance Matrices and Statistical Application, Acta Phys. Pol. B 36, 2681 (2005).

[13] F. Benaych-Georges and R. R. Nadakuditi, The Eigenvalues and Eigenvectors of Finite, Low Rank Perturbations of Large Random Matrices, Adv. Math. 227, 494 (2011).

[14] D. C. Hoyle and M. Rattray, Statistical Mechanics of Learning Multiple Orthogonal Signals: Asymptotic Theory and Fluctuation Effects, Phys. Rev. E 75, 016101 (2007).

[15] Z. Bai and J. W. Silverstein, Spectral Analysis of Large Dimensional Random Matrices, Springer Series in Statistics (Springer, New York, 2009), pp. 1-560.

[16] O. Ledoit and S. Péché, Eigenvectors of Some Large Sample Covariance Matrix Ensembles, Probab. Theory Relat. Fields 151, 233 (2011).

[17] N. Patterson, A. L. Price, and D. Reich, Population Structure and Eigenanalysis, PLoS Genet. 2, e190 (2006).

[18] G. McVean, A Genealogical Interpretation of Principal Components Analysis, PLoS Genet. 5, e1000686 (2009).

[19] A. L. Price, A. Helgason, S. Palsson, H. Stefansson, D. St. Clair, O. A. Andreassen, D. Reich, A. Kong, and K. Stefansson, The Impact of Divergence Time on the Nature of Population Structure: An Example from Iceland, PLoS Genet. 5, e1000505 (2009).
[20] G. J. Filion, J. G. van Bemmel, U. Braunschweig, W. Talhout, J. Kind, L. D. Ward, W. Brugman, I. J. de Castro, R. M. Kerkhoven, H. J. Bussemaker et al., Systematic Protein Location Mapping Reveals Five Principal Chromatin Types in Drosophila Cells, Cell 143, 212 (2010).

[21] J. Lachance, B. Vernot, C. C. Elbers, B. Ferwerda, A. Froment, J. M. Bodo, G. Lema, W. Fu, T. B. Nyambo, T. R. Rebbeck et al., Evolutionary History and Adaptation from High-Coverage Whole-Genome Sequences of Diverse African Hunter-Gatherers, Cell 150, 457 (2012).

[22] A. L. Price, A. Helgason, S. Palsson, H. Stefansson, D. St. Clair, O. A. Andreassen, D. Reich, A. Kong, and K. Stefansson, The Impact of Divergence Time on the Nature of Population Structure: An Example from Iceland, PLoS Genet. 5, e1000505 (2009).

[23] N. Halabi, O. Rivoire, S. Leibler, and R. Ranganathan, Protein Sectors: Evolutionary Units of Three-Dimensional Structure, Cell 138, 774 (2009).

[24] V. A. Marčenko and L. A. Pastur, Distribution of Eigenvalues for Some Sets of Random Matrices, Sb. Math. 1, 457 (1967).

[25] J. Baik, G. Ben Arous, and S. Péché, Phase Transition of the Largest Eigenvalue for Nonnull Complex Sample Covariance Matrices, Ann. Probab. 33, 1643 (2005).

[26] S. Péché, Universality Results for the Largest Eigenvalues of Some Sample Covariance Matrix Ensembles, Probab. Theory Relat. Fields 143, 481 (2009).

[27] B. Altshuler, H. Krovi, and J. Roland, Anderson Localization Makes Adiabatic Quantum Optimization Fail, Proc. Natl. Acad. Sci. U.S.A. 107, 12446 (2010).

[28] N. El Karoui, Concentration of Measure and Spectra of Random Matrices: Applications to Correlation Matrices, Elliptical Distributions and Beyond, Ann. Appl. Probab. 19, 2362 (2009).

[29] R. P. Feynman, Forces in Molecules, Phys. Rev. 56, 340 (1939).

[30] J. von Neumann and E. Wigner, Behavior of Eigenvalues in Adiabatic Processes, Z. Phys. 30, 467 (1929).

[31] J. W. Silverstein and Z. D. Bai, On the Empirical Distribution of Eigenvalues of a Class of Large-Dimensional Random Matrices, J. Multivariate Anal. 54, 175 (1995).

[32] N. El Karoui, Spectrum Estimation for Large Dimensional Covariance Matrices Using Random Matrix Theory, Ann. Stat. 36, 2757 (2008). 